\title{
Investigation of Optical Nonlinearities and Carrier Dynamics in In-Rich InGaN Alloys
}

\author{
S. NARgelas ${ }^{a *}$, T. MAlinauskas ${ }^{a}$, K. Jarasiunas ${ }^{a}$, \\ E. Dimakis ${ }^{b}$ And A. Georgakilas ${ }^{b}$ \\ ${ }^{a}$ Department of Semiconductor Optoelectronics \\ Institute of Materials Science and Applied Research, Vilnius University \\ Sauletekio Av. 9-3, LT-10222 Vilnius, Lithuania \\ ${ }^{b}$ 2Microelectronics Research Group, Physics Department, University of Crete \\ P.O. Box 2208, 71003 Heraklion, Greece \\ and IESL, FORTH, P.O. Box 1527, 71110 Heraklion, Greece \\ We present experimental studies of nonequilibrium carrier dynamics in \\ InGaN alloys with $70-90 \%$ content of In by using picosecond transient grat- \\ ing technique. The observed faster recombination rate in alloys with higher \\ Ga content and formation of a thermal grating via a lattice heating, being \\ more pronounced for layers with larger band gap, indicated that the main \\ reason of the heating is not the excess energy of photons, but the defect den- \\ sity which increases with Ga content. A gradual decrease in carrier lifetime \\ with excitation or with increasing temperature in 50-300 K range point out \\ the role of potential barriers in carrier recombination.
}

PACS numbers: 78.47.J-, 72.20.Jv

\section{Introduction}

InN and In-rich InGaN alloys are the least studied compounds of the IIInitride semiconductors. They attract particular interest due to lowest value of direct band gap and the highest electron mobility of this family materials, and their potential applications in near infrared optoelectronic devices. Nevertheless, the high background electron concentration and fast nonradiative recombination processes evidence that electrical properties are still not well controlled. Lattice mismatched heteroepitaxial growth leads to high density of extended defects, while the donor-like defects or impurites (e.g. nitrogen vacancies, oxygen and $\mathrm{Si}$ impurities, etc.) may also contribute to the formation of the nonradiative recombination centers [1].Therefore, measurement of carrier dynamics and understanding

*corresponding author; e-mail: saulius.nargelas@ff.vu.lt 
of their recombination mechanisms are important in optimizing materials growth conditions.

Up to now, a time-resolved differential transmission technique was mainly used for monitoring the carrier dynamics in InN at interlard excitation $(h \nu=$ $400 \mathrm{~nm}$ ) and probing at photoluminescence (PL) peak energy. An inverse relationship between carrier lifetime (varying from 50 ps to $500 \mathrm{ps)}$ ) and free electron concentration $\left(10^{18}\right.$ to $\left.10^{19} \mathrm{~cm}^{-3}\right)$ was reported and attributed to the trapping of photogenerated carriers by defects and impurities [1], while the carrier lifetime was found independent of the photogenerated carrier density, even when the latter was sufficiently higher than the free electron density. Moreover, the decreased carrier recombination rate at $20 \mathrm{~K}$ was attributed to radiative band-to-band transitions, assuming that nonradiative channels became non-active [2]. These assumptions need further verifications, as a role of extended defects in these studies was totally excluded. In this paper, we provide experimental studies of carrier dynamics and analysis of nonequilibrium processes in In-rich alloys in a wide temperature and excitation energy density range.

\section{Experimental}

$\mathrm{In}_{x} \mathrm{Ga}_{1-x} \mathrm{~N}$ heterostructures with In-content $x=0.7,0.8,0.9$ and free electron density in the range $(1-3) \times 10^{19} \mathrm{~cm}^{-3}$ were grown on $\mathrm{GaN} / \mathrm{Al}_{2} \mathrm{O}_{3}(0001)$ substrates by radio-frequency plasma-assisted molecular beam epitaxy. The different In-content was achieved by varying appropriately the $\mathrm{Ga}$ and $\mathrm{N}$ atomic fluxes while keeping In-flux constant. The growth temperature was chosen to be $400^{\circ} \mathrm{C}$, in order to avoid the decomposition of $\mathrm{InN}$ and to obtain, at the same time, an increased surface-diffusivity of the In-adatoms [3].

Time-resolved picosecond transient grating (TG) technique was used to study dynamics of nonequilibrium carriers. The interference pattern of two coherent $25 \mathrm{ps}$ duration laser pulses at $532 \mathrm{~nm}$ or $1064 \mathrm{~nm}$ wavelength was used to create a spatially modulated nonequilibrium carrier density $N(x)=N_{0}+\Delta N \cos (2 \pi x / \Lambda)$, resulting in a transient free carrier (FC) grating with a spacing $\Lambda$ and refractive index modulation $\Delta n(x, t) \propto \Delta N(x, t)$. A delayed probe pulse at 1064 or $1500 \mathrm{~nm}$ monitored the instantaneous diffraction efficiency of the grating $\eta(t)=I_{\mathrm{D}} / I_{\mathrm{T}}$ (here $I_{\mathrm{D}}$ is the diffracted and $I_{\mathrm{T}}$ is the transmitted probe beam parts), which is proportional to square of the index modulation, i.e. $\eta(t) \propto \Delta n^{2}(t)=\left[n_{\mathrm{eh}} \Delta N(t)\right]^{2}$. The kinetics of $\eta$ were measured at different excitation energies $\left(0.1\right.$ to $10 \mathrm{~mJ} \mathrm{~cm}^{-2}$ ), grating periods, and sample temperatures $(10-300 \mathrm{~K})$ and characterized by decay time $\tau_{\mathrm{G}}$.

\section{Results and discussion}

At $532 \mathrm{~nm}$ photoexcitation, the decreasing grating decay time indicated an increasing carrier recombination rate for alloys with higher Ga content. The 
longest lifetime with $\approx 200$ ps time was found in the layer with $90 \%$ of In. Moreover, the refractive index modulation by free carriers $\left(\Delta n_{\mathrm{FC}}<0\right)$ was competed by temperature at $\Delta n_{\mathrm{T}}>0$, and light diffraction on thermal grating determined the long-lasting decay component with the dip in diffraction kinetics at $\left|\Delta n_{\mathrm{FC}}\right|=\left|\Delta n_{\mathrm{T}}\right|$. The latter effect dominated in a layer with the largest band gap and pointed out that the main reason of the heating is not the excess energy of photons, but the rate of non-radiative recombination, being the fastest in the $\mathrm{In}_{0.7} \mathrm{Ga}_{0.3} \mathrm{~N}$ alloy.
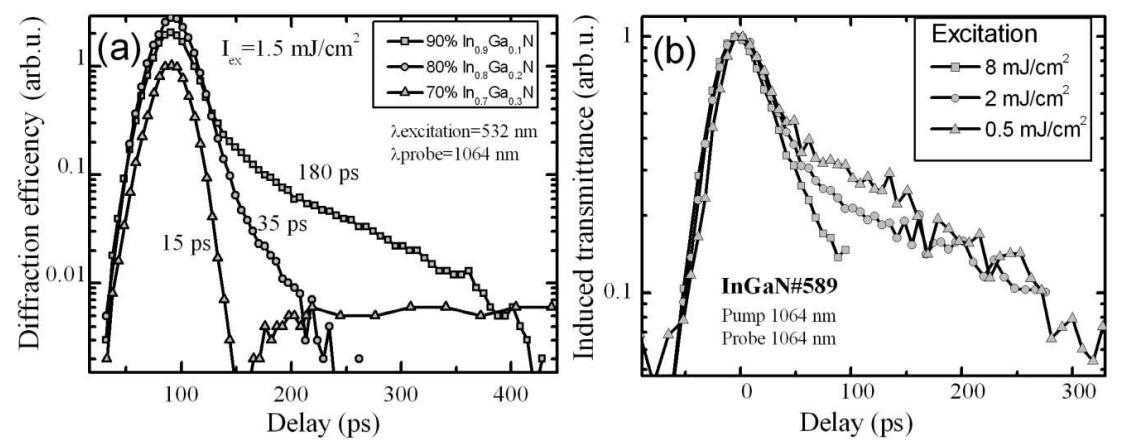

Fig. 1. Kinetics of FC grating with subsequent formation of a thermal grating (a) and absorption bleaching at excitation and probing in the vicinity of $E_{\mathrm{g}}(\mathrm{b})$.

At $1064 \mathrm{~nm}$ excitation and probing, we observed very strong absorption bleaching of the layers (up to $10 \%$ for $x=0.9$, see Fig. 1b). In the given case, the probe beam energy $(1.17 \mathrm{eV})$ was above the $E_{\mathrm{g}} \approx 0.9-1.0 \mathrm{eV}$ of the used InGaN alloys (for $x=0.8$ and 0.9 ), and thus controlled occupation of states in the bands. We attribute the bleaching to the dynamic filling of the higher states in the bands with subsequent relaxation to lower ones, occupation of which is driven by the carrier recombination. We note that the faster relaxation at higher excitations, seen as well in diffraction kinetics, points out to density-dependent recombination processes. In the final set of experiments, we tuned the probe beam energy of a parametric generator below the $E_{\mathrm{g}}$ of alloys (to $1.5-1.8 \mu \mathrm{m}$ ) while excitation was kept at $1064 \mathrm{~nm}$. This allowed us to probe only the FC concentration dynamics via $\Delta n_{\mathrm{FC}}$ modulation.

The measurements in a wide excitation range (Fig. 2) confirmed that recombination rate is weekly dependent on photogenerated carrier density and may lead to the thermal grating as well. The similar tendency of $\tau_{\mathrm{G}}(\Delta N)$ was found at $10 \mathrm{~K}$, while the $\tau_{\mathrm{G}}$ value increased and varied in range from 6 ns to $1.6 \mathrm{~ns}$ for

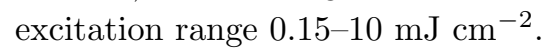

A set of grating decay kinetics have been measured at the same excitation conditions but varying the sample temperature (Fig. 3). We found that the grating decay rate is ruled by the thermal activation, and the determined value of 


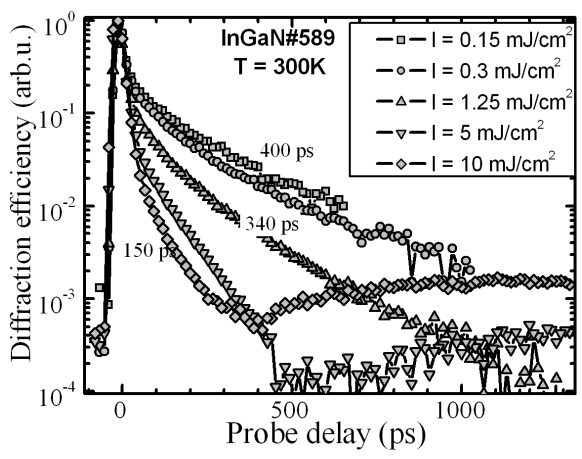

Fig. 2. Free carrier grating kinetics at probing below $E_{\mathrm{g}}$ with subsequent thermal grating effect at the highest excitations.
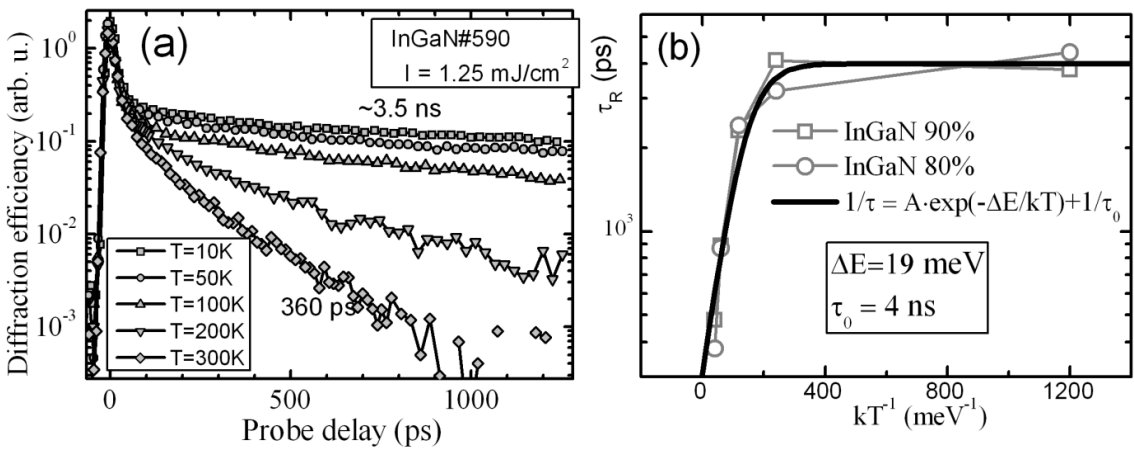

Fig. 3. Free carrier grating kinetics at different temperatures (a) and the dependence of grating decay time on inverse temperature (b).

$19 \mathrm{meV}$ (Fig. 3b) can be assumed as the activation energy for carrier recombination. Its origin may be ascribed to effects arising in the vicinity of dislocations [4], which are assumed to induce acceptor-like states, capture the free electrons, and become negatively charged. The space-charge region around dislocations leads to $E_{\mathrm{g}}$ modulation and spatial separation of the carriers, therefore photo- or thermal activation is needed for overcoming the local potential barrier and recombination. Indeed, dislocation related, $10 \mathrm{meV}$ barriers for photocurrent thermal activation were determined in conductive, non-intentionally doped GaN epilayers [5]. As the radius of the perturbed region approximately equals to the Debye length, $\lambda_{\mathrm{D}}=\left(\varepsilon \varepsilon_{0} k T / e^{2} n\right)^{1 / 2}$, both temperature and illumination may influence the effective area around dislocations and modify the electrical activity of the charged dislocations. The Debye radius decreases at lower temperatures, making the averaged contribution of dislocations as fast recombination centers less efficient. This effect may explain the observed monotonous increase in carrier lifetime with cooling. On the other hand, in high excitation range, when nonequilibrium carrier density approaches the equilibrium one, a screening of the barriers may enhance 
the contribution of dislocations to recombination. This tendency was also observed in our experiments at various temperatures, and points out that the role of extended defects must be considered as well.

The thermal behavior of PL intensity provided a similar value of the activation energy, $7.8 \mathrm{meV}$, in InN [6]. Assuming that the activation energy separates the regions of radiative and nonradiative recombination, the plateau value of carrier lifetime, $t_{0}=4 \mathrm{~ns}$ at $10 \mathrm{~K}$ might be already influenced by the radiative recombination [2]. Nevertheless, the weak dependence of $\tau_{\mathrm{G}}$ versus excitation at low $T$ points out that radiative recombination is still masked by defects, which is in agreement with PL data analysis [6]. Therefore, further studies of radiative and nonradiative recombination mechanisms in InGaN alloys and $\mathrm{InN}$ require layers with lower free electron density.

\section{Acknowledgments}

The work was partly supported by the Lithuanian State Science and Studies Foundation and EC Project "SELITEC" (contract No. G5MA-CT-2002-04047).

\section{References}

[1] F. Chen, A.N. Cartwright, H. Lu, W.J. Schaff, J. Cryst. Growth 269, 10 (2004).

[2] F. Chen, A.N. Cartwright, W.J. Schaff, Phys. Status Solidi A 202, 768 (2005).

[3] E. Dimakis, E. Iliopoulos, K. Tsagaraki, A. Georgakilas, Appl. Phys. Lett. 86, 133104 (2005).

[4] J.-L. Farvacque, Z. Bougrioua, I. Moerman, Phys. Rev. B 63, 15202 (2001).

[5] Z. Bougrioua, M. Azize, B. Beaumont, P. Gibart, T. Malinauskas, K. Neimontas, A. Mekys, J. Storasta, K. Jarasiunas, J. Cryst. Growth 300, 228 (2007).

[6] R. Intartaglia, B. Maleyre, S. Ruffenach, O. Briot, T. Taliercio, B. Gil, Appl. Phys. Lett. 86, 142104 (2005). 